\title{
Feline Immunodeficiency Virus Gag Is a Nuclear Shuttling Protein
}

\author{
Iris Kemler, ${ }^{a}$ Dyana Saenz, ${ }^{a}$ and Eric Poeschla ${ }^{a, b}$ \\ Department of Molecular Medicine, and Division of Infectious Diseases, ${ }^{\text {b }}$ Mayo Clinic College of Medicine, Rochester, Minnesota, USA
}

Lentiviral genomic RNAs are encapsidated by the viral Gag protein during virion assembly. The intracellular location of the initial Gag-RNA interaction is unknown. We previously observed feline immunodeficiency virus (FIV) Gag accumulating at the nuclear envelope during live-cell imaging, which suggested that trafficking of human immunodeficiency virus type 1 (HIV-1) and FIV Gag may differ. Here we analyzed the nucleocytoplasmic transport properties of both Gag proteins. We discovered that inhibition of the CRM1 nuclear export pathway with leptomycin B causes FIV Gag but not HIV-1 Gag to accumulate in the nucleus. Virtually all FIV Gag rapidly became intranuclear when the CRM1 export pathway was blocked, implying that most if not all FIV Gag normally undergoes nuclear cycling. In FIV-infected feline cells, some intranuclear Gag was detected in the steady state without leptomycin B treatment. When expressed individually, the FIV matrix (MA), capsid (CA), and nucleocapsid-p2 (NC-p2) domains were not capable of mediating leptomycin B-sensitive nuclear export of a fluorescent protein. In contrast, CANC-p2 did mediate nuclear export, with MA being dispensable. We conclude that HIV-1 and FIV Gag differ strikingly in a key intracellular trafficking property. FIV Gag is a nuclear shuttling protein that utilizes the CRM1 nuclear export pathway, while HIV-1 Gag is excluded from the nucleus. These findings expand the spectrum of lentiviral Gag behaviors and raise the possibility that FIV genome encapsidation may initiate in the nucleus.

G ag is both necessary and sufficient for retrovirus particle formation and budding. The protein is translated on free polysomes and targeted to the plasma membrane, where virus particles are assembled (16). A central question is whether Gag and the viral genomic RNA (gRNA) associate at the plasma membrane or earlier during assembly and, if so, whether this occurs in the cytosol; in association with organelles, e.g., cotranslationally; or even in the nucleus, as was described previously for an alpharetrovirus (17). Human immunodeficiency virus type 1 (HIV-1) gRNAs become anchored at the plasma membrane before particle assembly is detectable, but it is not clear whether the Gag-gRNA complexes of this and other lentiviruses first form in the cytoplasm and then transit to the plasma membrane or the gRNA traffics there independently $(26,27)$. Biochemical experiments supported the former scenario, with a monomer or low-order multimers forming on HIV-1 gRNAs and higher-order multimer formation depending on subsequent plasma membrane interactions (33).

Gag is encoded by the full-length unspliced viral RNA. To circumvent the cellular checkpoint that prevents the export of intron-containing mRNA, lentiviruses express Rev, which functions as an adaptor between the cellular karyopherin CRM1/exportin-1 and a Rev response element (RRE) located in the $3^{\prime}$ region of unspliced viral RNAs (9). However, the main cellular function of the CRM1 nuclear export pathway is the export of cellular proteins containing a nuclear export signal (NES) as well as ribosomal subunits, $5 \mathrm{~S}$ ribosomal RNAs and uridylate-rich small nuclear RNAs (UsnRNAs) (14). In contrast, most cellular mRNAs are exported from the nucleus via NXF1/NXT. This pathway is exploited by the simple orthoretrovirus MasonPfizer monkey virus (MMPV), which has a constitutive RNA transport element (CTE) that recruits $\operatorname{NXF1}$ directly $(4,19)$.

The trafficking itinerary and assembly competence of HIV-1 Gag can be influenced by the nuclear export history of the mRNA from which it is translated $(1,25,52)$. If the HIV-1 RRE is replaced by the MPMV CTE, the normally Rev-dependent unspliced RNA exits the nucleus efficiently, but Gag translation is inefficient (7).
Replacement of the RRE by the hepatitis B virus posttranscriptional regulatory element (PRE) results in normal Gag expression but reduces the budding efficiency by 10 -fold (24). Cell- and species-specific effects have also been observed. For example, Gag expressed from a Rev-dependent RNA is assembly incompetent in some rodent cell lines, but replacing of the RRE with the CTE restores assembly (52).

We recently used protein and RNA labeling in live cells to show that feline immunodeficiency virus (FIV) Gag and gRNA accumulate, independently of each other, at the nuclear envelope (30). Discrete foci of colocalized FIV Gag and gRNA were also observed at the cytoplasmic surface of the nuclear envelope. In contrast, and consistent with observations reported previously by many laboratories, we never saw HIV-1 Gag colocalize with the nuclear envelope. While HIV-1 gRNA was detectable there, this was observed much less frequently than with FIV gRNA, and it was not seen in most cells (30). For both lentiviruses, gRNA was visualized at the plasma membrane only if Gag was coexpressed and the gRNA packaging signal was intact. Here we pursued these observations further by determining whether either lentiviral Gag protein accesses the intranuclear compartment. The results reveal a surprising, sharp difference between the two lentiviruses.

\section{MATERIALS AND METHODS}

Plasmids, cells, transfections. Cyan fluorescent protein (CFP) refers to the enhanced version (eCFP). pFIVGag-CFP (also termed pFP93gagCFP) and pHIVGag-CFP were described previously (30). pFIVGag-CFP is derived from the FIV packaging plasmid pFP93 (44) (a diagram of pFP93 is

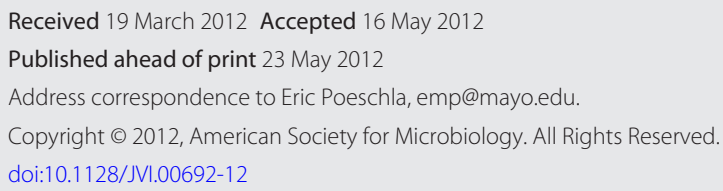


A CMVP 1 sd \begin{tabular}{c|c|c|}
\hline gag & CFP \\
\hline
\end{tabular}
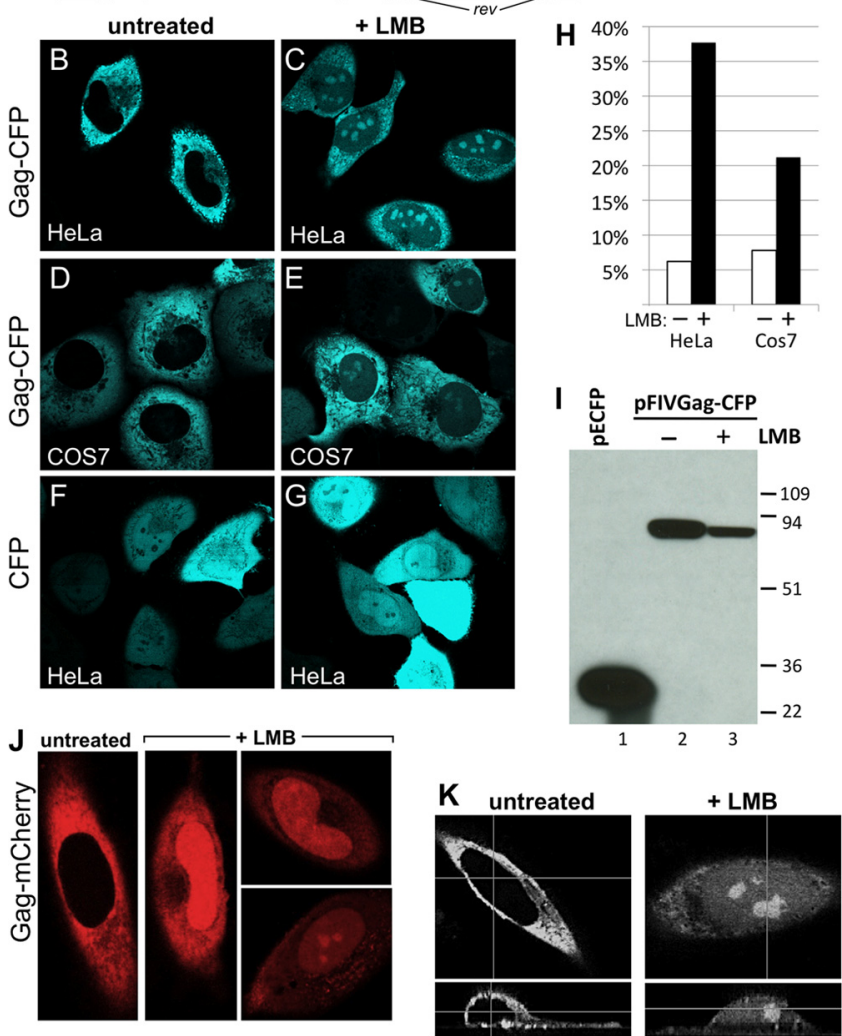

FIG 1 Subcellular localizations of FIV Gag-CFP and Gag-mCherry with and without LMB treatment. (A) pFIVGag-CFP expresses Gag-CFP and Rev. The encapsidation signal is deleted. sd, major splice donor; ires-pac, nonretroviral stuffer DNA. (B to G) Live-cell confocal images of HeLa (B, C, F, and G) or COS7 (D and E) cells transfected with pFIVGag-CFP (B to E) or pCFP (F and $\mathrm{G}$ ) and either left untreated (B, D, and F) or treated with $10 \mathrm{ng} / \mathrm{ml} \mathrm{LMB}$ for 3 $\mathrm{h}(\mathrm{C}, \mathrm{E}$, and $\mathrm{G})$. The intranuclear foci seen in panels $\mathrm{C}$ and $\mathrm{E}$ were verified to be nucleoli by costaining with an antibody against the nucleolar marker fibrillarin (data not shown). (H) The MFI was measured in the nucleus and cytoplasm of 38 cells $(5,6,12$, and 15 cells for the columns going from left to right, respectively), and the ratio of the MFI in the nucleus to the MFI in the total cell was determined and is expressed as a percentage (mean \pm standard deviation). (I) HeLa cells were transfected with pCFP (lane 1) or pFIVGag-CFP (lanes 2 and 3 ), and cells were either untreated (lanes 1 and 2) or treated with LMB (10 $\mathrm{ng} / \mathrm{ml}$ ) for $3 \mathrm{~h}$. Cell lysates were separated on a $10 \%$ SDS-PAGE gel and probed with primary mouse anti-CFP and secondary HRP-conjugated goat antimouse antibodies. The lesser band intensity in lane 3 is likely to reflect a reduced delivery of Rev-dependent Gag-CFP mRNA to the cytoplasm in the presence of LMB (14). (J) Imaging, as described above for panels B to G, with pFPGag-mCherry instead of pFPGag-CFP. (K) $z$ stack for FIV Gag-CFP. Images are representative of more than six independent experiments.

provided in Fig. 4A), which lacks the encapsidation signal and cis-acting elements needed for reverse transcription and integration; Env, Vif, and OrfA are not encoded, and most of the env gene between the two rev exons is replaced by a nonretroviral sequence, an internal ribosome entry sitepuromycin resistance gene cassette (ires-pac) (Fig. 1A). pFIVGag $\Delta$ MACFP, pFIVGag $\Delta$ CA-CFP, pFIVGag $\Delta$ NCp2-CFP, pFIV-MA-CFP, pFIVCA-CFP, and pFIV-NCp2-CFP, used to study domain contributions and assess potential FIV Gag nuclear localization signals (NLSs) and nuclear export signals (NESs), were made by standard PCR-based mutagenesis, using pFIVGag-CFP as a template. pFP93 $\mathrm{Pr}^{\mathrm{m}}$ was constructed by overlap extension PCR that introduced an Asp-to-Gly change in the LLDTG motif (35) in the protease active site of pFP93 (the D30 GAC codon was changed to GAG). The deletion of pol from pFP93 (to yield pFP93 $\Delta \mathrm{Pol}$ ) was achieved with an EcoRI-XcaI adaptor that was ligated between the EcoRI site near the gag C terminus and an XcaI site located at nucleotide (nt) 232 of the integrase gene. This eliminated all of pol except for the 614-nt integrase gene 3' remnant; therefore, Gag but no pol-encoded viral enzymes were expressed. Correct plasmid constructions were all confirmed by sequencing.

pCT5 (cytomegalovirus [CMV] promoter fused to the FIV R repeat at TATA box plasmid $\underline{5}$ ) is used to produce infectious, replication-competent FIV in human cells, which is enabled because the human cell-inactive $5^{\prime}$ FIV U3 element was replaced with the human CMV (hCMV) promoter just upstream of the $5^{\prime} \mathrm{R}$ repeat $(41,42)$. pCT5 was derived from the infectious 34TF10 molecular clone previously isolated from a lambda phage library by Talbott et al. (53). Note that pCT5 encodes the wild-type (WT), normally infectious $34 \mathrm{TF} 10$ virus, since it expresses the full $34 \mathrm{TF} 10$ R-to-R proviral transcript with a normal 5' cap site and a normal 3' U3 $(41,42)$. pCT5efs is identical to pCT5 except for a 31-nt insertion that causes an envelope frameshift in the central region of env; pCT5efs was used to produce vesicular stomatitis virus glycoprotein G (VSV-G)-pseudotyped, single-cycle, full-length FIV (29).

CMVDR8.9 expresses HIV-1 Gag-Pol, Tat, and Rev (56). NL4-3R-E$\Delta 426$, also referred to here and in our previous papers as HIV- $1_{\text {luc }}$, is a single-cycle HIV-1 luciferase reporter virus (36); its provirus differs from HIV-1 NL4-3 in only three ways: it has a 426-nt deletion in env, a frameshift in $v p r$, and a replacement of nef with the firefly luciferase gene (luc). Pseudotyped $\Delta e n v$ FIV 34 TF10 (expressed by pCT5efs) and HIV-1 $1_{\text {luc }}$ were produced in $293 \mathrm{~T}$ cells as previously described (44).

Crandell feline kidney (CrFK) (8), 293T, HeLa, and COS7 cells were cultured in Dulbecco's modified Eagle's medium with $10 \%$ fetal calf serum. DNA transfection was carried out with calcium phosphate coprecipitation (293T, HeLa, and CrFK cells) or Fugene 6 (Roche Applied Sciences) (COS7 cells). Titers of FIV were determined by the quantification of infectious foci at $48 \mathrm{~h}$ in CrFK cells using the immunofluorescence method described below.

Indirect immunofluorescence, live-cell imaging, and immunoblotting. HeLa cells were transfected with $2 \mu \mathrm{g}$ of plasmid or transduced with VSV-G-pseudotyped CT5efs or VSV-G-pseudotyped HIV-1 luc $_{\text {. Cells were }}$ either left untreated or treated with leptomycin B (LMB; Sigma-Aldrich) at $10 \mathrm{ng} / \mathrm{ml}(18 \mathrm{nM})$ for $3 \mathrm{~h}$. A CRM1-dependent green fluorescent protein (GFP) shuttle indicator, Rev1.4-GFP-NES, containing the NES from the adenomatous polyposis coli tumor suppressor protein, was used as a positive control for the LMB blockade of the CRM1 pathway (23). For indirect immunofluorescence, cells were fixed with 3\% paraformaldehyde for $15 \mathrm{~min}$ on ice, washed with phosphate-buffered saline (PBS), quenched with $50 \mathrm{mM} \mathrm{NH}_{4} \mathrm{Cl}$ for $10 \mathrm{~min}$, washed with PBS, permeabilized with $0.2 \%$ Triton X-100 for $10 \mathrm{~min}$ at room temperature, washed, and blocked with $3 \%$ low-fat milk for $30 \mathrm{~min}$. Cells were then incubated with monoclonal antibody (MAb) PAK3-2C1 (1:200; Custom Monoclonal Antibodies International, Sacramento, CA), which recognizes FIV capsid, or with monoclonal antibody $183-\mathrm{H} 12-5 \mathrm{C}$ to HIV-1 p24, from the NIH AIDS Reference and Reagent Program, at a 1:4,000 dilution overnight at room temperature in a humidified chamber. For fibrillarin, a primary rabbit antibody (Novus Biologicals) was used at a 1:200 dilution. After cells were washed with PBS, they were blocked again and then incubated with an anti-mouse Alexa Fluor 594- or anti-rabbit Alexa Fluor 488-conjugated secondary antibody (1:1,000; Molecular Probes) for 30 min, washed, and mounted in Prolong Gold (Molecular Probes). Slides were analyzed on a Zeiss LSM 510 confocal laser scanning microscope equipped with an Axiovert $200 \mathrm{M}$ inverted platform using a Plan-Apochromat $100 \times$ (1.4-numerical-aperture) oil immersion objective. For live-cell imaging, HeLa cells or COS7 cells were seeded into Lab-Tek glassbottom chamber slides (Nunc) and observed at $20 \mathrm{~h}$ posttransfection with the above-described Zeiss LSM 510 confocal laser scanning microscope. CFP was excited with an argon laser at $458 \mathrm{~nm}$, and emission was detected with a band-pass filter of 475 to $525 \mathrm{~nm}$. The quantification of the nuclear translocation of FIV Gag-CFP in HeLa and COS7 cells was done with Zeiss 
KS400 image analysis software. The mean fluorescence intensity (MFI) was measured in the nucleus or the cytoplasm, and the area of the two compartments was determined. The total MFI was determined by multiplying the MFI with the area: $\%=$ total MFI (nucleus)/total MFI (nucleus + cytoplasm).

For immunoblotting, cells were lysed in $1 \times$ radioimmunoprecipitation assay (RIPA) buffer $(150 \mathrm{mM} \mathrm{NaCl}, 1 \% \mathrm{NP}-40,0.5 \%$ deoxycholate, $0.1 \%$ SDS, $50 \mathrm{mM}$ Tris $[\mathrm{pH} 8.0]$ ) containing protease inhibitors (Complete Mini; Roche). Fifteen micrograms of cell lysate was separated on a reducing 10\% SDS-polyacrylamide gel and transferred onto a polyvinylidene difluoride membrane (Immobilon-P; Millipore). The membranes were blocked in $0.2 \%$ I-block (Tropix)- $0.1 \%$ Tween and then probed with a monoclonal anti-GFP/CFP antibody (1:5,000; BD Biosciences), referred to below as the anti-CFP antibody. As a secondary antibody, a horseradish peroxidase (HRP)-conjugated goat anti-mouse antibody (1:1,000; Calbiochem) was used. Bands were visualized with Lumi-Light detection reagent (Roche).

\section{RESULTS}

Nuclear import and CRM1-dependent export of FIV Gag-CFP. To study subcellular localization in living cells, we fused CFP to the FIV Gag C terminus (pFIVGag-CFP) (Fig. 1A). As we previously observed (30), in HeLa or COS7 cells, FIV Gag-CFP has a mostly dispersed but nonhomogeneous cytoplasmic distribution (Fig. 1B and D). However, when cells were incubated for $3 \mathrm{~h}$ with leptomycin B (LMB), a specific inhibitor of CRM1-mediated nuclear export (14), Gag-CFP accumulated in the nucleus (Fig. 1C and $\mathrm{E}$ ). In contrast, CFP was distributed as expected between the nucleus and cytoplasm and was unaffected by LMB (Fig. 1F and $\mathrm{G})$. The nuclear accumulation of Gag-CFP was partial, with an apparent majority of the protein remaining in the cytoplasm. In both cell types, Gag-CFP also localized in nucleoli, while CFP did not (Fig. 1C, E, and K).

To quantify LMB-induced nuclear translocation, the mean fluorescence intensity (MFI) in the nucleus and the cytoplasm was measured, and the area of the compartments was determined. The total MFI was calculated (MFI $\times$ area) for the nucleus and the cytoplasm, and the ratio of the total MFI of the nucleus to the total MFI of the cell (nucleus plus cytoplasm) was determined (Fig. $1 \mathrm{H})$. In untreated HeLa cells, $6.2 \%$ of the total fluorescence was nuclear. In LMB-treated cells, however, the nuclear proportion increased 6-fold, to $37.7 \%$. In COS7 cells, the fluorescence intensity increased 2.7 -fold, from $7.8 \%$ for untreated cells to $21.2 \%$ with LMB treatment. To exclude the possibility that this nuclear localization represented the import of an LMB-induced proteolytic cleavage fragment of Gag-CFP, Western blots of HeLa cell lysates were probed with an antibody to CFP. As demonstrated in Fig. 1I, FIV Gag-CFP remained intact in the presence and absence of LMB, with neither free CFP nor any other CFP-containing fragment being generated. Therefore, the nuclear fluorescence observed in Fig. 1C and $\mathrm{E}$ is due to the nuclear trafficking of fulllength Gag-CFP, and the apparent restriction to the cytoplasm in the steady state in untreated cells requires CRM1-mediated export. We next imaged, in a similar fashion, FIV Gag fused to a strictly monomeric fluorescent protein, mCherry (Fig. 1J). The use of mCherry resulted in an increased nuclear versus cytoplasmic localization in the presence of LMB (Fig. 1J). Nucleolar concentration was also evident in some cells, although this was less frequent than with CFP as the fusion partner ( $<20 \%$ of cells). $z$ stacks of FIV Gag-CFP-expressing cells confirmed a lack of nuclear localization in the steady state and abundant nuclear accu-

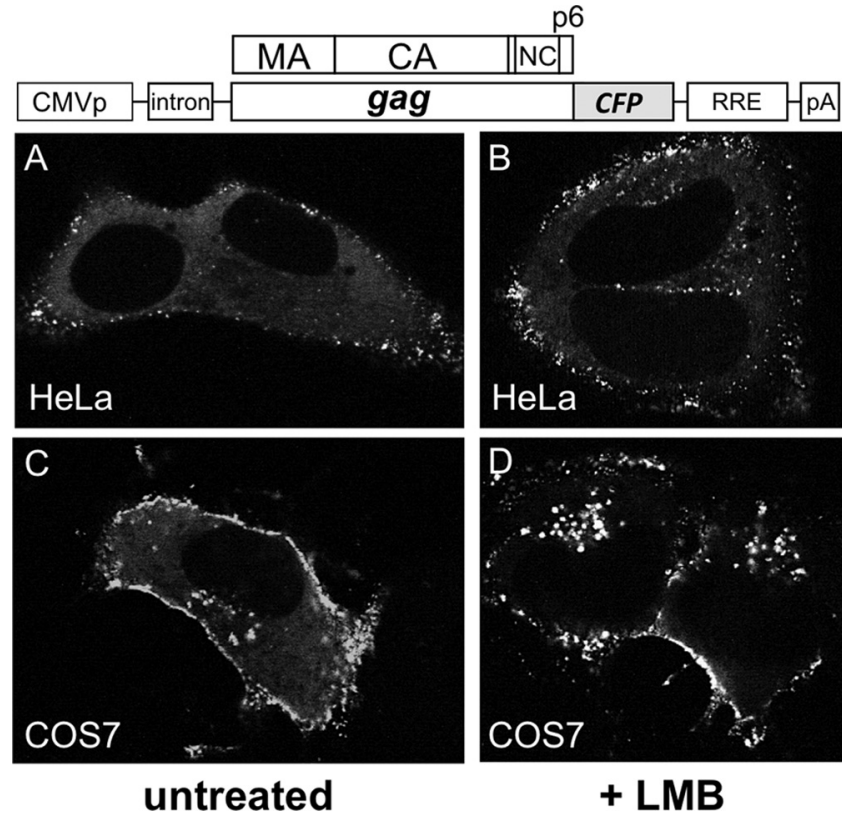

FIG 2 Subcellular localization of HIV-1 Gag-CFP with and without LMB Shown are live-cell confocal images of HeLa cells (A and B) or COS7 cells (C and D) transfected with pHIVGag-CFP $(1 \mu \mathrm{g})$ (plasmid diagrammed at the top) and pCMV $\Delta$ R8.91 $(0.5 \mu \mathrm{g})$. The cells were left untreated (A and C) or were treated with $\mathrm{LMB}(\mathrm{B}$ and $\mathrm{D})(10 \mathrm{ng} / \mathrm{ml})$ for $3 \mathrm{~h}$. Images are representative of at least four independent experiments.

mulation with a nucleolar concentration after LMB treatment (Fig. 1K). We readdress nucleolar localization below.

HIV-1 Gag-CFP does not traffic to the nucleus. To establish a comparative benchmark for these CRM1 export pathway-dependent effects, we analyzed the subcellular localization of HIV-1 Gag-CFP in parallel (Fig. 2). HIV-1 Gag-CFP exhibited a cytoplasmic localization with punctate foci as well as a diffuse signal in both cell types and strong plasma membrane accumulation in COS7 cells. Similarly to FIV Gag-CFP, HIV-1 Gag-CFP was excluded from nuclei. However, in clear contrast to FIV Gag-CFP, LMB treatment did not result in nuclear HIV-1 Gag-CFP accumulation. No difference in the subcellular distributions of HIV-1 Gag-CFP was observed for LMB-treated versus untreated cells.

Nuclear import and retention of unlabeled FIV Gag but not HIV-1 Gag. In order to exclude the possibility that the CFP or mCherry moiety causes an aberrant nuclear translocation of FIV Gag, we determined the trafficking of the unmodified Gag proteins of HIV-1 and FIV in the presence and absence of LMB. In addition, we employed both virus infection and DNA transfection in these experiments (Fig. 3).

Cells were first transfected with standard FIV and HIV-1 vector packaging plasmids that produce Gag and Gag-Pol polyproteins from Rev-dependent RNAs. Indirect immunofluorescence was performed with antibodies to FIV capsid or HIV-1 capsid. Again, as seen with FIV Gag-CFP, a pronounced nuclear retention of FIV Gag was observed upon LMB treatment (Fig. 3A and B). When the amounts of nuclear accumulation were compared, even more native FIV Gag than FIV Gag-CFP was nuclear (compare Fig. 1C, E, $\mathrm{J}$, and $\mathrm{K}$ with $3 \mathrm{~B}$ ). The nucleolar concentration, although consistently detectable, was much less common than with the Gag-CFP fusion, varying from 1 to $10 \%$ of cells in multiple experiments. In 

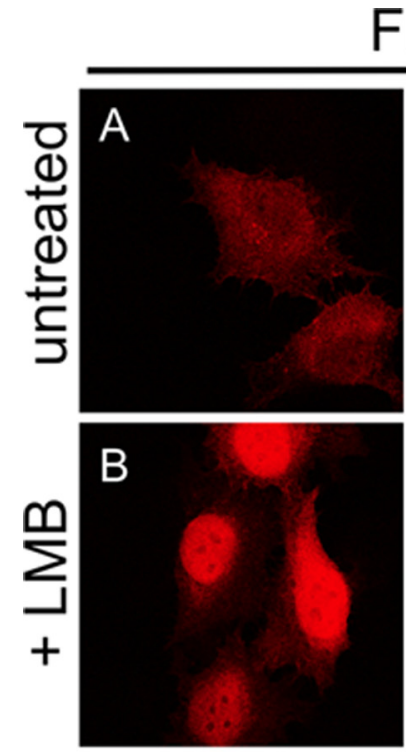

transfected
FIV
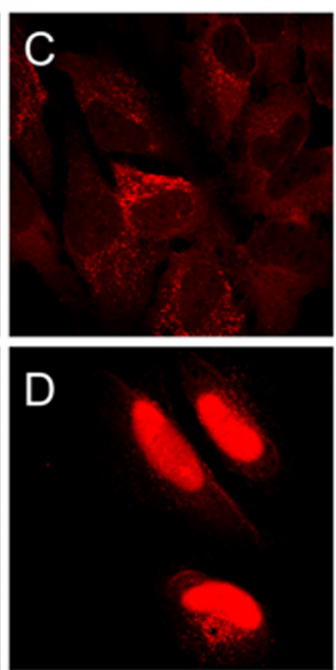

infected
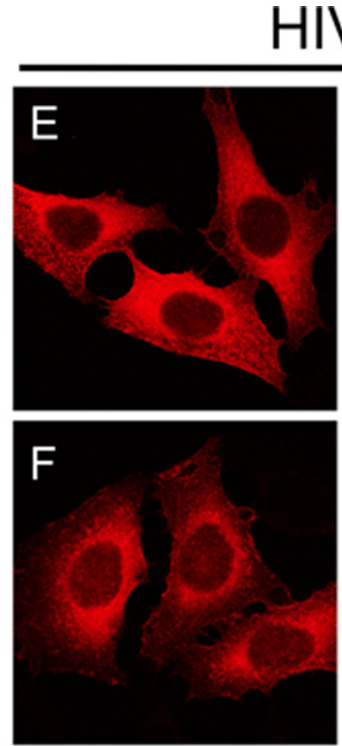

transfected
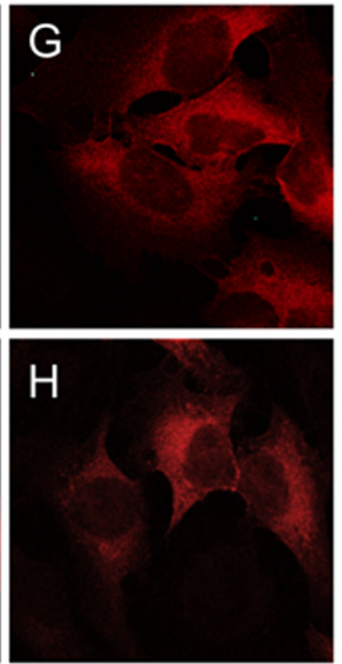

infected
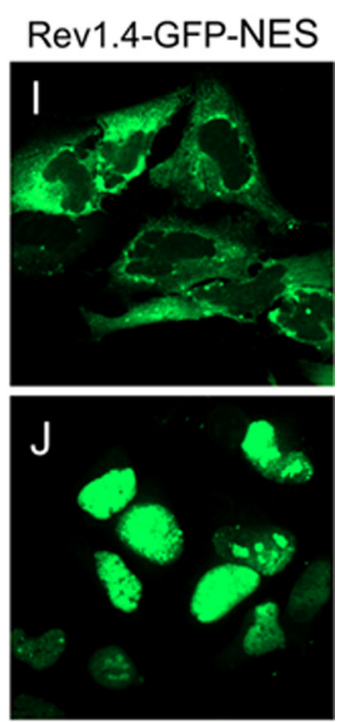

transfected

FIG 3 Subcellular localization of unmodified Gag proteins analyzed in transfected and infected cells. (A to H) Both FIV (A to D) and HIV-1 (E to H) were tested. For transfections, HeLa cells were transfected with either FIV packaging plasmid pFP93 (A and B) or HIV-1 packaging plasmid CMVDR8.91 (E and F). Alternatively, cells were infected with single-cycle VSV-G-pseudotyped env-lacking viruses for FIV infection (C and D) and HIV-1 infection (G and H). These single-cycle challenge viruses were generated in $293 \mathrm{~T}$ cells by pseudotyping the viruses produced by pCT5efs and pHIV-1 $1_{\text {luc }}$, respectively. (Note that pCT5efs, used here [C and D] and in feline cells in Fig. 6B produces FIV 34TF10, which is entirely of the wild type except for a 31-bp oligonucleotide inserted into env to frameshift it and allow single-cycle pseudotyping [29]). Forty-eight hours after transfection or infection, cells were left untreated or were treated with LMB for $3 \mathrm{~h}$ and then fixed, and indirect immunofluorescence was performed with monoclonal anti-FIV CA or monoclonal anti-HIV CA antibody and an anti-mouse Alexa Fluor 594-conjugated secondary antibody. (I and J) A CRM1-dependent shuttle protein, Rev1.4-GFP-NES (23), was used as a positive control. Images are representative of at least three independent experiments for each virus.

clear contrast to FIV Gag, LMB did not result in a nuclear translocation of unlabeled HIV-1 Gag (Fig. 3E and F).

We then carried out viral infection rather than transfection to confirm these results and verify that they occur in the normal context of Gag expression from integrated proviruses of infected cells. To closely mimic the viral life cycle through a single round of infection and assembly, we began by infecting HeLa cells with VSV-G-pseudotyped FIV and HIV-1 particles containing envframeshifted but otherwise full-length genomes. After chromosomal integration, each lentivirus carries out the normal production of spliced and unspliced mRNAs and Gag/Gag-Pol production. Here again, FIV Gag accumulated in the nucleus after LMB treatment (Fig. 3C and D), but HIV-1 Gag did not and remained in the cytoplasm (Fig. $3 \mathrm{G}$ and $\mathrm{H}$ ). The dramatic difference between FIV and HIV-1 is clear by comparing Fig. 3D and H. Also note that, in contrast to FIV Gag-CFP, virtually all native FIV Gag is imported into the nucleus (Fig. $3 \mathrm{~B}$ and $\mathrm{D}$ ), phenocopying a positive-control LMB-dependent shuttle protein in this regard (Fig. 3I and J). This does not represent any general compromise of the nuclear envelope barrier after LMB treatment, because the experiments contain an ideal "control" protein of the same molecular mass and domain structure (HIV-1 Gag). Thus, the steady state in these cells represents the vigorous reexport of FIV Gag, which becomes important in experiments described below.

Concomitantly with or shortly after budding, the Gag precursor p55 is cleaved by the virally encoded protease (PR) (28). To verify that the increase in nuclear fluorescence was due to the transport of intact Gag and not of the CA subdomain detected by the monoclonal antibody, we mutated the critical aspartic acid at position 30 of the protease catalytic domain (35) to a glycine residue in the FIV vector packaging plasmid pFP93 (44), generating pFP93Pr ${ }^{\mathrm{m}}$ (Fig. 4A). A pol-deleted version that lacks all of the protease gene and the rest of pol except for a fragment of the integrase reading frame was also made (FP93 $\Delta \mathrm{pol})$. Western blotting showed that the cleavage of p55 Gag was abolished for both FP93Pr ${ }^{\mathrm{m}}$ and FP93 $\Delta$ pol (Fig. 4A [the blot also reconfirms, this time using antibody to Gag rather than to CFP as in Fig. 1I, that Gag-CFP does not fragment]). pFP93 $\mathrm{Pr}^{\mathrm{m}}$ and $\mathrm{pFP} 93 \Delta \mathrm{Pol}$ were transfected into HeLa cells, and immunofluorescence analysis was performed. LMB treatment resulted in the nuclear accumulation of both protease mutant FIV Gag polyproteins, similarly to Gag expressed from wild-type pFP93 (Fig. 4B). Moreover, the same results were also found when polyclonal anti-FIV sera from infected cats were used (data not shown).

In summary, HIV-1 Gag does not cycle through the nucleus. In stark contrast, experiments with Gag-CFP and with unlabeled Gag produced from either transfected plasmids or an integrated provirus demonstrate that FIV Gag transiently cycles through the nucleus, where it is retained when CRM1-mediated export is prevented.

CTE- versus Rev-mediated Gag mRNA export. How viral RNA traffics out of the nucleus and into the cytoplasm can influence the trafficking itinerary of the Gag protein translated from the RNA and modulate the efficiency of assembly $(1,51)$. We therefore investigated if changing the FIV RNA export pathway from a Rev/RRE-dependent mechanism to a CTE-dependent pathway affects the nuclear trafficking capability of FIV Gag-CFP. The FIV RRE and rev exons were replaced with four copies of the 

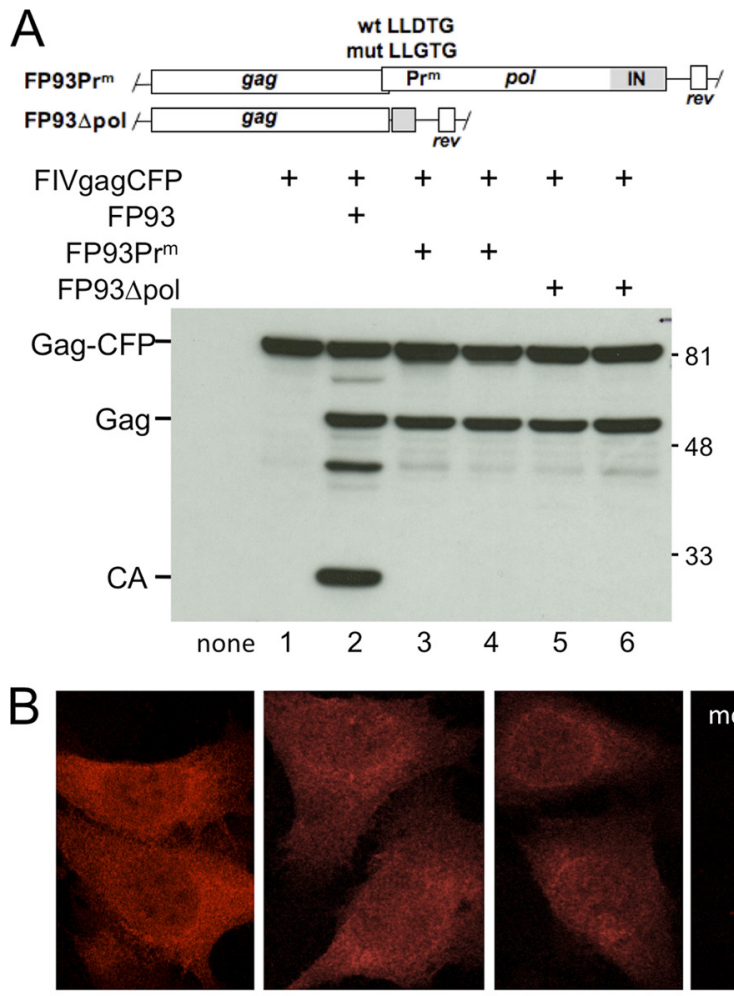

mock
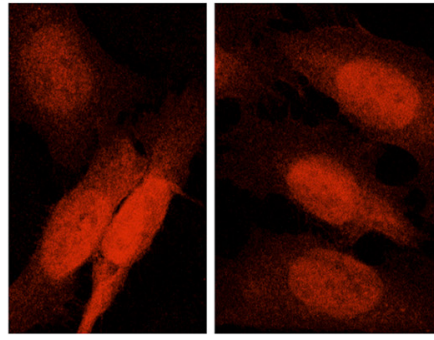

FP93

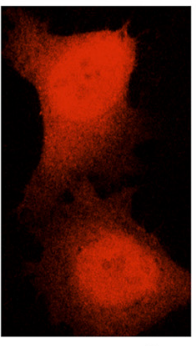

$\mathrm{FP93} \triangle \mathrm{Pol}$

FIG 4 Subcellular localization of FIV Gag polyprotein. (A) pFP93Pr ${ }^{\mathrm{m}}$ (protease catalytic domain mutant) and pFP93 $\Delta \mathrm{Pol}$ (lacks pol) are illustrated (the segments containing gag-pol and the first exon of rev are shown). The bottom shows immunoblotting for these constructs along with pFIVgagCFP; in lane 2, pFP93, which encodes Gag-Pol (protease intact), was included. None indicates that no Gag DNA was transfected. (B) Indirect immunofluorescence showing subcellular distribution with and without LMB treatment. MAb PAK3-2C1, which recognizes an epitope in the CA domain of Gag, was the primary antibody $(\mathrm{Ab})$ for immunofluorescence and immunoblotting. Mock-transfected cells (top) or pFP93-transfected cells incubated only with anti-mouse Alexa Fluor 594-conjugated secondary antibody (bottom) are shown at the right. Images are representative of at least three independent experiments.

constitutive transport element (CTE) from MPMV, resulting in pFIVGag-CFP4xCTE (Fig. 5). The FIV Gag-CFP thus generated was excluded from the nucleus (Fig. 5, left). LMB treatment resulted in nuclear and nucleolar translocations indistinguishable from those observed when FIV Gag-CFP was produced from a Rev-dependent RNA (compare Fig. 5, right, with 1C, E, J, and K). We conclude that the nuclear export pathway of the RNA does not determine the ability of FIV Gag to cycle through the nucleus.

Feline cells display FIV Gag nuclear shuttling, and infected cells contain nuclear Gag in the steady state. Cells from different mammalian species display various competences for specific lentiviral life cycle stages. Impediments arise from species-specific
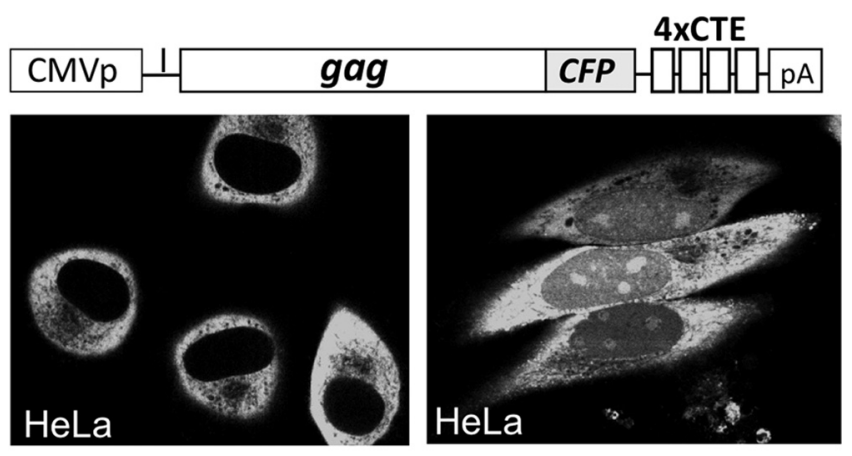

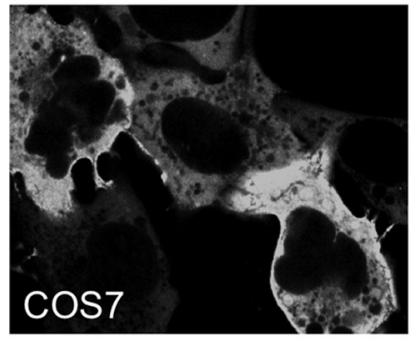

untreated

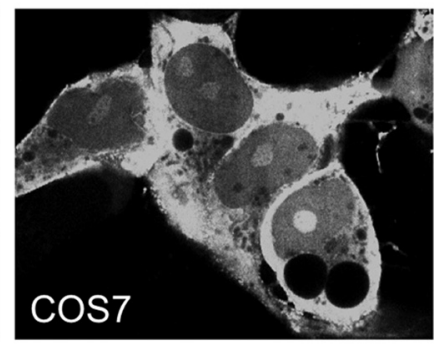

$+\mathrm{LMB}$
FIG 5 Export of Gag-CFP mRNA via the NFX1/NXT pathway does not alter CRM1-dependent nuclear cycling of Gag-CFP. Diagrammed at the top is pFIVGag-CFP-4xCTE, which encodes the FIV Gag-CFP fusion protein and in which the RRE and Rev exons of FIV are replaced with four copies of the CTE of MPMV. Photomicrographs show live-cell confocal images of HeLa or COS7 cells transfected with pFIVGag-CFP-4xCTE and treated or not with LMB for $3 \mathrm{~h}$. Images are representative of at least three independent experiments.

variations in restriction and dependency factors. The latter phenomenon is particularly evident in the case of HIV-1 gRNA trafficking and particle assembly in mouse cells, which proceed inefficiently $(3,38,52)$. Indeed, variant residues in murine CRM1 itself have been reported to account for a substantial portion of this assembly-side block (50). We therefore considered that FIV Gag nuclear trafficking in human and African green monkey cells might proceed differently than in cells of the natural feline host. To test this possibility, we carried out transfection and infection of Crandell feline kidney (CrFK) cells. FIV Gag-CFP and unmodified FIV Gag were imaged by using direct excitation and immunofluorescence, respectively (Fig. 6).

This experiment not only demonstrated that FIV Gag cycles through the nucleus in feline cells but also showed that a substantial portion of FIV Gag can be seen to also accumulate in the nucleus when the CRM1 pathway is uninhibited (Fig. 6 and 7A). As in the human cell experiments (Fig. 3B and D and 4B), native Gag protein nuclear residence was nearly total after LMB treatment. Note that Fig. 6C shows feline cells that are productively infected at a low multiplicity of infection (MOI) (0.3) with wildtype, replication-competent FIV. Here the same LMB-induced nuclear concentration of Gag was seen (Fig. 6C). The representative syncytia shown demonstrate the approximately equivalent nuclear and cytoplasmic FIV Gag levels in the steady state (no LMB), while nuclear levels were much higher than cytoplasmic levels in infected cells after LMB treatment (Fig. 6C).

Nucleolar localization. As noted above, the FIV Gag-CFP protein consistently displayed nucleolar localization in most LMBtreated cells, while unmodified FIV Gag assessed by confocal im- 
A
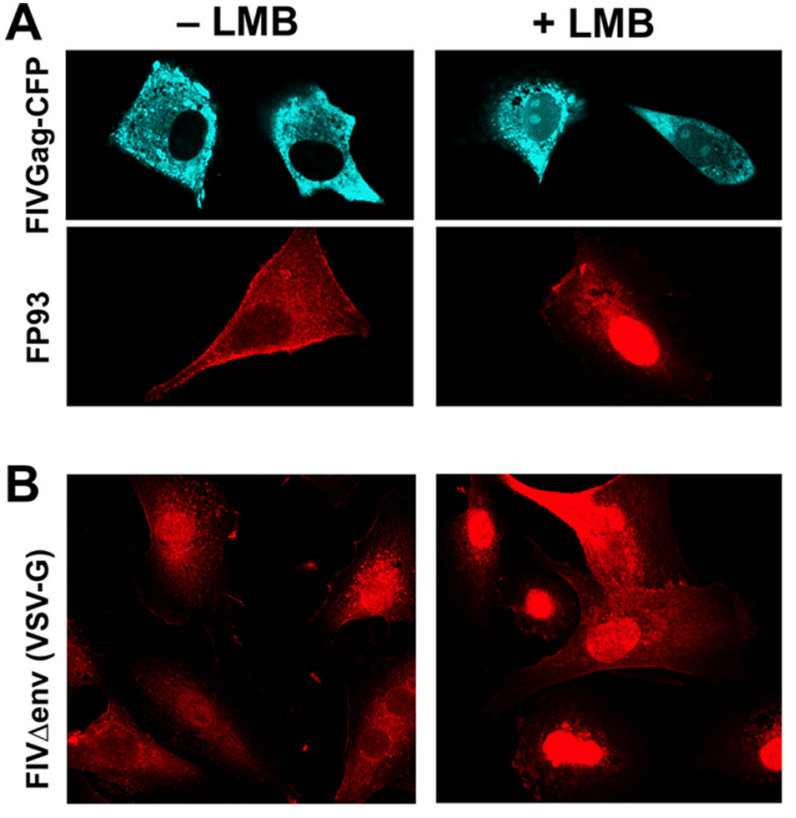

C - LMB

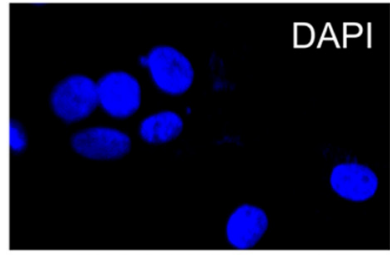

Gag
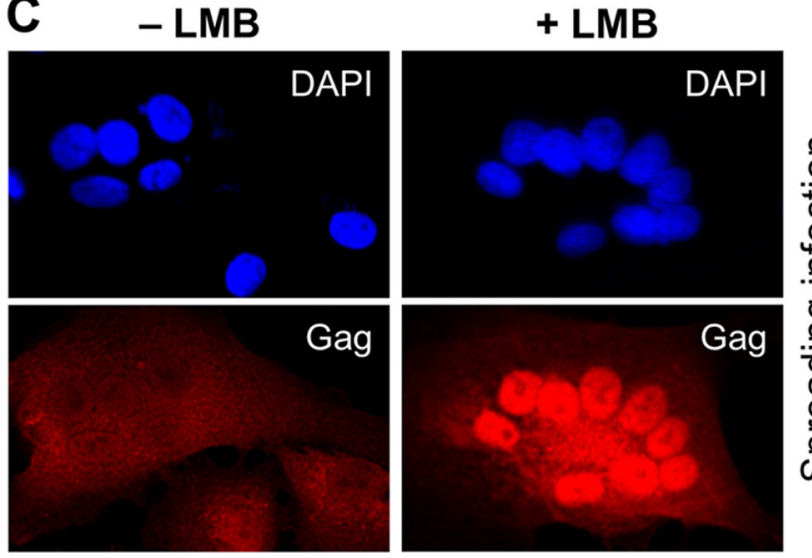

FIG 6 FIV Gag nuclear cycling in feline cells. (A) CrFK cells were transfected with expression plasmids. (B) CrFK cells were infected at an MOI of 5 with VSV-G-pseudotyped, env-frameshifted FIV generated with pCT5efs. (C) CrFK cells were infected with wild-type, replication-competent FIV at an MOI of 0.3 . DAPI (4',6-diamidino-2-phenylindole) was used to identify nuclei; 6and 8-cell syncytia are seen at the left and right, respectively. Fluorescence was imaged directly in panel $\mathrm{A}$, while indirect immunofluorescence with $\mathrm{MAb}$ PAK3-2C1 was performed $48 \mathrm{~h}$ after infection in panels B and C. LMB treatment was done for $2 \mathrm{~h}$. Images are representative of the appearance of cells in at least three independent experiments for panels $\mathrm{A}$ and $\mathrm{B}$ and two independent experiments for panel C.

munofluorescence displayed a clearly visible nucleolar location less frequently (ranging from 1 to $10 \%$ of cells in multiple experiments). To address the phenomenon of nucleolar targeting further and confirm the identity of the structures, we performed simultaneous immunofluorescence experiments with unmodified FIV Gag and the nucleolar marker fibrillarin in FIV-infected cells (Fig. 7A), pFP93-transfected cells (Fig. 7B), and pFP93 $\Delta$ poltransfected cells (Fig. 7C). Fibrillarin, an rRNA 2' -O-methyltransferase, is associated with the U3, U8, and U13 small nuclear RNAs and is located in the dense fibrillar component (DFC) of the nucleolus. Some Gag was visible in the nuclei of FIV-infected cells in
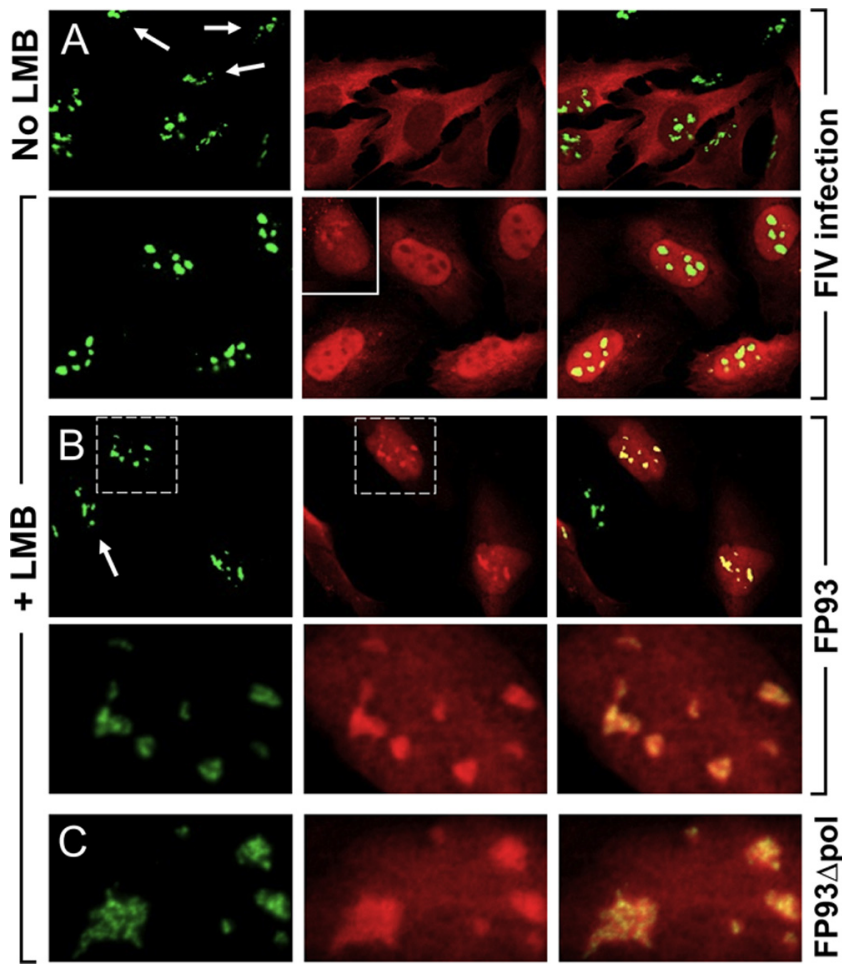

FIG 7 FIV Gag and fibrillarin coimaging of FIV-infected cells and in FIV Gag-Pol- and Gag-transfected cells. Confocal immunofluorescence was performed in HeLa cells with an antibody to the nucleolar marker fibrillarin (green) and Gag/CA-recognizing MAb PAK3-2C1 (red). (A) Cells infected with VSV-G-pseudotyped FIV $(\Delta e n v)$ at an MOI of 5 and treated or not with LMB. Images are representative of the majority (>90\%) of infected cells. However, some nucleolar localization of Gag was also seen in 5 to $10 \%$ of LMBtreated infected cells (small inset photomicrograph at the top left in the middle panel). Staining controls with MAb PAK3-2C1 showed no background in uninfected and untransfected cells (not shown); specificity was also established by the uninfected/untransfected cells that stained for fibrillarin but not at all for Gag (white arrows in panels A and B). (B and C) FIV Gag-Pol (B) or Gag $\Delta$ pol (C) was expressed by plasmid transfection using pFP93 and pFP93 $\Delta$ pol, respectively. The bottom row in panel B shows enlarged views of the nucleus within the dashed boxes.

the absence of LMB (Fig. 7A); however, nucleolar concentration of Gag was observed infrequently. LMB again induced a major shift to nuclear-retained Gag (Fig. 7A). A nucleolar concentration was seen in a small and variable percentage of infected LMBtreated cells ( 5 to $10 \%$ ), but in most infected cells, nucleoli had less Gag than the surrounding nucleoplasm (Fig. 7A, compare the cell in the inset photomicrograph with other cells). In cells transfected with pFP93 or pFP93 $\Delta$ pol, the nucleolar Gag concentration was more evident. In some transfections, such cells comprised 20 to $30 \%$ of the transfected monolayer, which may be related to the higher Gag expression levels achieved with transfection (Fig. 7B and C). In such cells, Gag was diffusely distributed within the nucleoli, with the signal not strictly confined to the fibrillarinmarked DFC (Fig. 7).

Assessment of potential nuclear localization and export signals. Since FIV Gag enters the nucleus, where it is retained if the CRM1 nuclear export pathway is inhibited, we hypothesized that it may contain classical nuclear localization and export signals. An analysis of the p55 Gag amino acid sequence with the Eukaryotic Linear Motif resource (11) revealed a potential leucine-rich NES 
in the MA subdomain between amino acids 53 and 66 (DIPETLD QLRLVIC). However, mutations of the three leucines and the isoleucine at position 65 to alanines (DIPETADDQARAVAC) did not result in a nuclear accumulation of Gag (data not shown), and therefore, this sequence does not function as an NES. We also identified a possible bipartite NLS in MA $\left[\mathrm{KR}\left(\mathrm{X}_{10}\right) \mathrm{KSKK}\right]$ with the cNLS Mapper program (31). Mutations of the four lysines and the arginine to alanines did not prevent nuclear import after LMB treatment, and therefore, this motif is not a functional NLS (data not shown). To investigate other potential signals, we constructed pFIVGag-CFP mutants (Fig. 8). These mutants lack MA ( $\triangle \mathrm{MA}$ $\mathrm{CFP}), \mathrm{CA}(\Delta \mathrm{CA}-\mathrm{CFP})$, or NCp2 $(\Delta \mathrm{NCp} 2-\mathrm{CFP})$. Live-cell confocal images are shown in Fig. 8A to $\mathrm{H}$, and the ratio of nuclear/total MFI is shown in Fig. 8J. These experiments showed that Gag $\Delta M A-$ CFP behaved most similarly to Gag-CFP in the steady state, being present only in scant amounts in the nucleus in some cells and absent from the nucleus in others (Fig. 8A and C). This nuclear accumulation increased strongly after LMB treatment, although the intranuclear appearance differed somewhat from that of GagCFP (Fig. 8D and J). The CA deletion mutant at steady state approximately phenocopied the LMB-treated WT protein (compare Fig. $8 \mathrm{~B}$ with $\mathrm{E}$ and $\mathrm{F}$ ). Importantly, no further increase in the nuclear accumulation of the $\triangle \mathrm{CA}$ protein was observed with LMB (Fig. 8E, F, and J). The subcellular localization of the protein lacking NCp2 was similarly indistinguishable in the presence or absence of LMB (Fig. 8G, H, and J). The NCp2-deleted protein distributed between the cytoplasm and the nucleus but was excluded from nucleoli. It was also indistinguishable from CFP alone (Fig. $1 \mathrm{~F}$ and $\mathrm{G}$ and $8 \mathrm{G}$ and $\mathrm{H}$ ). Thus, FIV NCp2 contains the nucleolartargeting determinant of Gag-CFP.

To investigate this further, we analyzed individual Gag domains as CFP fusion proteins (Fig. 8K to Q). MA-CFP was diffusely present in both the cytoplasm and nucleus and was unaffected by LMB (Fig. 8K, L, and Q). CA-CFP localized to the cytoplasm and nucleus but was excluded from nucleoli, a distribution similar to those seen with MA-CA-CFP (i.e., $\triangle \mathrm{NCp} 2-\mathrm{CFP}$ ) and CFP alone (compare Fig. 8M, N, and Q with Fig. 8G, H, and J, and Fig. $1 F$ and G). Suggesting that when it is expressed outside the viral context in this manner, CA is passively distributed, like CFP. NCp2-CFP showed a pronounced nuclear accumulation. $\mathrm{NCp} 2$ was also the only one of the three proteins to confer an accumulation of signal in nucleoli, confirming that it has the determinant for nucleolar targeting (Fig. $8 \mathrm{O}$ and $\mathrm{P}$ ). Note also that this nucleolar targeting is infrequent with the full-length FIV Gag protein unless it is fused to CFP (compare Fig. $1 \mathrm{C}$ and $\mathrm{E}$ and $8 \mathrm{~A}$ and $\mathrm{B}$ with $3 \mathrm{~B}$ and $\mathrm{D}$ and $7 \mathrm{~A}$ ). There was no increase in the nuclear proportion of any of the three individually expressed Gag domains (MA-CFP, CA-CFP, or NCp2-CFP) upon LMB treatment (Fig. 8Q).

We conclude that each tested FIV Gag domain can enter the nucleus. Since no further increase in nuclear accumulation was observed when the CRM1 export pathway was inhibited, we also conclude that none of the individual domains demonstrates a classical NES. Importantly, CA-NCp2, CA, and NCp2 each differ markedly in the nucleocytoplasmic trafficking properties that they confer to a fluorescent protein (compare Fig. 8C and D with M and $\mathrm{N}$ and with $\mathrm{O}$ and $\mathrm{P}$ ). Likewise, FIV Gag proteins lacking either CA or NCp2 did not retain the LMB-sensitive nuclear export properties of full-length Gag (Fig. 8E to H). A consideration of CA-NCp2 (i.e., $\triangle \mathrm{MA}$ ) in the context of all of the data shown in
Fig. 1 to 8 indicates that the CRM1-dependent nuclear shuttling of FIV Gag is an emergent property of the combined CA-NCp2 portion that is not manifested by CA or NCp2 alone and which is unlikely to be mediated by a single linear peptide motif unless that motif is functionally unmasked in the context of the larger protein.

\section{DISCUSSION}

Our main discovery in this report, that a lentiviral Gag protein cycles through the nucleus, was unexpected. The HIV-1 MA domain was reported previously to have two NLSs $(5,21)$ and one NES (13), but multiple subsequent reports have shown that HIV-1 MA-GFP is excluded from the nucleus $(2,10)$ and that it does not contain a conventional NLS $(15,22)$. Two studies in the past year confirmed that HIV-1 Gag lacks a CRM1-dependent nuclear export signal $(2,18)$. In addition, HIV-1 Gag lacking the reported nuclear import and export signals can mediate efficient packaging (18). Our present results with HIV-1 Gag support the current consensus that a nuclear function of this protein in assembly can be excluded unless this occurs at a level that is below the detection limits of present methodologies. FIV is thus unique among the lentiviruses in having a Gag protein that undergoes nuclear cycling. In addition to expanding the spectrum of lentiviral Gag behaviors, these results raise the possibility that FIV genome encapsidation initiates in the nucleus. A comparative analysis of FIV and HIV-1 Gag/gRNA trafficking may help illuminate the spatial and temporal details of the encapsidation process in the cell.

There are two interesting precedents for retroviral Gag nuclear trafficking, both in nonlentiviruses. Parent and colleagues have shown in a series of studies that Rous sarcoma virus (RSV) Gag shuttles $(6,17,20,45-47)$, and experiments with RSV Gag mutants that do not undergo nuclear cycling provided evidence that this is needed for efficient gRNA encapsidation $(17,45)$. RSV Gag nuclear import is mediated by NLSs in MA and NC (45). In a recent study, the replacement of RSV MA with HIV-1 MA prevented detectable nuclear cycling of the resultant Gag chimera (2). The formation of infectious virus, as assessed by the ratio of transduced to transfected cells in a reporter virus plasmid-transfected monolayer, was not blocked, although it was reduced 50 to $70 \%$ (2). The binding of RSV Gag to the gRNA in the nucleus exposes an NES in the Gag p10 domain, which in turn promotes CRM1RanGTP binding, which results in the nuclear export of the complex $(20,45,47)$. The nuclear encapsidation process of this alpharetrovirus so far remains unique among retroviruses (40).

Prototype foamy virus (PFV) Gag has also been observed in the nuclear compartment $(39,48,55)$. The mechanism is novel, since PFV Gag does not possess a functional NLS and does not translocate to the nucleus of interphase cells. Instead, it accesses the nucleus during mitosis and binds to the host cell chromatin, which is mediated by a chromatin binding site (CBS) in the NC domain (39). Recently, an NES in PFV Gag was described (43). The role of Gag nuclear trafficking in the PFV life cycle remains uncertain, with nuclear gRNA encapsidation not yet implicated.

Not only does FIV Gag undergo nuclear cycling, we observed that it can also be partially nuclear at the steady state when the CRM1 export pathway is not inhibited (Fig. 4B, 6B and C, and 7A) and also that virtually complete nuclear accumulation occurs in both human and feline cells when the CRM1 export pathway is inhibited (Fig. 3A to F, 4B, and 6C to F). The results suggest that 

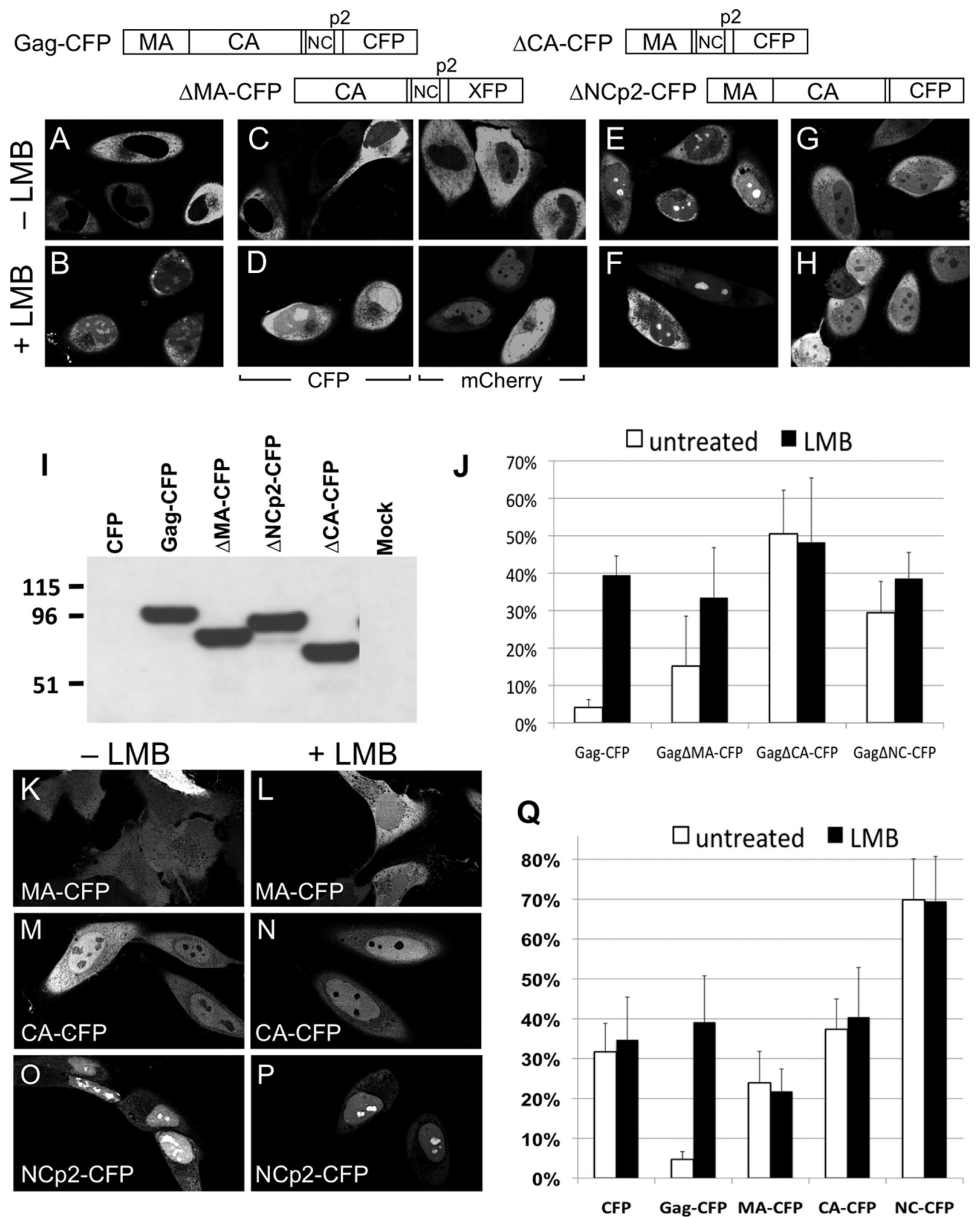

FIG 8 Subcellular localization of FIV Gag mutants. (A to H) Live-cell confocal images of HeLa cells transfected with $2 \mu \mathrm{g}$ of pFIVGag-CFP (A and B), pFIVGag $\Delta$ MA-CFP (left) or pFIVGag $\Delta$ MA-mCherry (right) (C and D) pFIVGag $\Delta$ CA-CFP (E and F), or pFIVGag $\Delta$ NC-CFP (G and H) and treated or not with LMB for $3 \mathrm{~h}$. (I) Immunoblotting confirmed the expression of single proteins of the predicted masses. The control (rightmost) lane was moved for presentation purposes by using editing software. (J) The MFI was measured in the nucleus and cytoplasm of 137 cells $(10,8,18,21,25,11,18$, and 26 cells for the columns going from left to right, respectively), and the ratio of the MFI in the nucleus to the MFI in the total cell was determined and expressed as a percentage (mean \pm standard deviation). (K to Q) Subcellular localization of FIV MA-CFP, CA-CFP, and NCp2-CFP. Transfected HeLa cells were treated or not with LMB for $3 \mathrm{~h}$. The nuclear/total MFI (Q) was determined as described above for panel J for 158 cells $(6,17,25,23,15,15,21,16,11$, and 9 cells for columns from left to right, respectively). Images are representative of at least three independent experiments. 
most, if not all, FIV Gag normally undergoes a nuclear cycle. The individual Gag domain-CFP fusions that we tested did not accumulate further in the nucleus with LMB treatment, and therefore none demonstrated a classical CRM1-dependent NES when tested in this manner. In contrast, LMB-sensitive nuclear export is conferred by CA-NCp2 but not by CA or NCp2 alone, and Gag deletants that lack either CA or NCp2 lose the nuclear export properties of full-length Gag. The nuclear concentration of CA-NCp2CFP and CA-NCp2-mCherry in the presence of LMB (Fig. 8C and D) also implies that they are actively imported into the nucleus. Three general mechanisms can be considered. The first is that FIV Gag is a cargo that CRM1 engages by recognizing individually insufficient nuclear export determinants present in separate Gag domains (a multipartite signal). Such an export mechanism has been identified, for example, in Snurportin-1. Snurportin-1 binds CRM1 in a bipartite manner, with a leucine-rich NES located in the $\mathrm{N}$ terminus and a second signal in a basic surface on the nucleotide binding domain (12). The second possibility is that CANCp2 and/or the full-length Gag protein forms or exposes one or more noncanonical determinants that are not formed by individually expressed MA, CA, or NCp2. A third possibility would be a more indirect mechanism whereby Gag associates with another factor or macromolecular complex that shuttles. FIV p2 comprises the C-terminal 18 amino acids of Gag and contains the PSAP (late-domain) motif. p2 mediates Tsg101 interactions and particle release from the plasma membrane, analogously to HIV-1 p6 (37). While it remains possible that p2 contributes to fulllength Gag nuclear cycling, it did not display such properties in the context of NCp2 (Fig. $8 \mathrm{O}$ and P) or $\Delta \mathrm{CA}$ (Fig. 8E and F).

Note that fusion to FIV CA does not appreciably alter the nucleocytoplasmic distribution of CFP (compare Fig. 8M and N with $1 F$ and $G$ ). These studies, in which CA was artificially expressed as a discrete protein, do not, of course, exclude that core-associated CA derived from Gag cleavage during particle budding has important nuclear import functions in the postentry virion. Although the present work focused on the trafficking of de novo-expressed Gag, which is relevant to the assembly side of the life cycle, there is evidence that FIV and HIV-1 negotiate early events differently in some respects, including the use of nuclear entry pathways $(32,34$, 54). Further comparative investigations of the roles of FIV versus HIV-1 Gag-derived virion structural proteins in preintegration trafficking steps may be informative.

The nucleolar targeting that we observed is of unclear significance. It is evidently dependent on NC, but the various ways in which this did or did not manifest in the various proteins that we tested may have implications. NC-conferred nucleolar localization was evident in multiple protein contexts (Fig. 1, 5, 7, and 8). The full-length Gag nucleolar concentration that became detectable (i.e., by reaching concentrations higher than those in the surrounding nucleoplasm) could be seen with all expression modes (virus infection, plasmid transfection, unmodified Gag, fluorescent protein-fused Gag, and in the presence and absence of functional protease). However, it was less frequent with Gag than with Gag-CFP. Fusion to mCherry, which is monomeric (49), produced reduced nucleolar localization compared to that of CFP. These observations, along with the fact that in LMB-treated cells, the fusion proteins are not fully nuclear, while unmodified FIV Gag is, suggest that the fusion of proteins to the C terminus of FIV Gag can modulate pertinent functions. This is an important area for further investigation.
When considered in the context of the effects of LMB, the steady-state accumulations of FIV Gag on the outer face of the nuclear envelope that we observed previously (30) are consistent with a model in which the CRM1-mediated FIV Gag nuclear export rate exceeds the Gag import rate. When expressed abundantly, FIV Gag may become relatively traffic jammed at the entry side, yielding a steady-state appearance with no visible nuclear Gag. Since fluorescent protein fusions to the FIV Gag C terminus appear to reduce its nuclear import, as a comparison of Fig. 1, 5, and $6 \mathrm{~A}$ with 3,4 , and $6 \mathrm{~B}$ and $\mathrm{C}$ in the present work shows, they may elevate this outer face stacking to a detectable level.

In summary, we demonstrate that FIV Gag is a nuclear shuttling protein that utilizes the CRM1 nuclear export pathway. We show this with transfected and infected cells, with fluorescent protein-fused Gag and native Gag, with human and feline cells, and under conditions where the presence of single full-length Gag proteins was verified. The experiments at the same time bolster the current consensus in refuting the notion of a nuclear phase of HIV-1 Gag. Our results raise the possibility that FIV encapsidation initiates in the nucleus, as in the prevailing RSV model. Because Gag is the driver of particle assembly, during which it participates in numerous sequential processes and protein-protein interactions, determining the specific role of Gag trafficking patterns in gRNA encapsidation remains technically challenging but represents an exciting future direction. The clear difference in nuclear cycling between HIV-1 and FIV Gag may provide utility for a comparative attack on the major unsolved problem of deciphering the spatiotemporal specifics of lentiviral gRNA encapsidation in the cell.

\section{ACKNOWLEDGMENTS}

We thank T. Rinkoski and M. Peretz for technical assistance, J. Tarara in the Mayo Clinic Flow Cytometry and Imaging Core for help with confocal microscopy and image analysis software, and C. Grant of Custom Monoclonal Antibodies International for the gift of MAb PAK3-2C1.

\section{REFERENCES}

1. Balasubramaniam M, Freed EO. 2011. New insights into HIV assembly and trafficking. Physiology (Bethesda) 26:236-251.

2. Baluyot MF, Grosse SA, Lyddon TD, Janaka SK, Johnson MC. 2012. CRM1-dependent trafficking of retroviral Gag proteins revisited. J. Virol. 86:4696-4700.

3. Bieniasz PD, Cullen BR. 2000. Multiple blocks to human immunodeficiency virus type 1 replication in rodent cells. J. Virol. 74:9868-9877.

4. Bray M, et al. 1994. A small element from the Mason-Pfizer monkey virus genome makes human immunodeficiency virus type 1 expression and replication Rev-independent. Proc. Natl. Acad. Sci. U. S. A. 91:12561260.

5. Bukrinsky MI, et al. 1993. A nuclear localization signal within HIV-1 matrix protein that governs infection of non-dividing cells. Nature 365: 666-669.

6. Butterfield-Gerson KL, Scheifele LZ, Ryan EP, Hopper AK, Parent LJ. 2006. Importin-beta family members mediate alpharetrovirus gag nuclear entry via interactions with matrix and nucleocapsid. J. Virol. 80:17981806.

7. Coyle JH, et al. 2003. Sam68 enhances the cytoplasmic utilization of intron-containing RNA and is functionally regulated by the nuclear kinase Sik/BRK. Mol. Cell. Biol. 23:92-103.

8. Crandell RA, Fabricant CG, Nelson-Rees WA. 1973. Development, characterization, and viral susceptibility of a feline (Felis catus) renal cell line (CRFK). In Vitro 9:176-185.

9. Cullen BR. 2003. Nuclear mRNA export: insights from virology. Trends Biochem. Sci. 28:419-424.

10. Depienne C, et al. 2000. Cellular distribution and karyophilic properties of matrix, integrase, and Vpr proteins from the human and simian immunodeficiency viruses. Exp. Cell Res. 260:387-395. 
11. Dinkel H, et al. 2012. ELM - the database of eukaryotic linear motifs. Nucleic Acids Res. 40:D242-D251. doi:10.1093/nar/gkr1064.

12. Dong X, et al. 2009. Structural basis for leucine-rich nuclear export signal recognition by CRM1. Nature 458:1136-1141.

13. Dupont S, et al. 1999. A novel nuclear export activity in HIV-1 matrix protein required for viral replication. Nature 402:681-685.

14. Fornerod M, Ohno M, Yoshida M, Mattaj IW. 1997. CRM1 is an export receptor for leucine-rich nuclear export signals. Cell 90:1051-1060.

15. Fouchier RA, Meyer BE, Simon JH, Fischer U, Malim MH. 1997. HIV-1 infection of non-dividing cells: evidence that the amino-terminal basic region of the viral matrix protein is important for Gag processing but not for post-entry nuclear import. EMBO J. 16:4531-4539.

16. Freed EO. 1998. HIV-1 gag proteins: diverse functions in the virus life cycle. Virology 251:1-15.

17. Garbitt-Hirst R, Kenney SP, Parent LJ. 2009. Genetic evidence for a connection between Rous sarcoma virus gag nuclear trafficking and genomic RNA packaging. J. Virol. 83:6790-6797.

18. Grewe B, et al. 2012. Cytoplasmic utilization of human immunodeficiency virus type 1 genomic RNA is not dependent on a nuclear interaction with gag. J. Virol. 86:2990-3002.

19. Gruter P, et al. 1998. TAP, the human homolog of Mex67p, mediates CTE-dependent RNA export from the nucleus. Mol. Cell 1:649-659.

20. Gudleski N, Flanagan JM, Ryan EP, Bewley MC, Parent LJ. 2010. Directionality of nucleocytoplasmic transport of the retroviral gag protein depends on sequential binding of karyopherins and viral RNA. Proc. Natl. Acad. Sci. U. S. A. 107:9358-9363.

21. Haffar OK, et al. 2000. Two nuclear localization signals in the HIV-1 matrix protein regulate nuclear import of the HIV-1 pre-integration complex. J. Mol. Biol. 299:359-368.

22. Hearps AC, Wagstaff KM, Piller SC, Jans DA. 2008. The N-terminal basic domain of the HIV-1 matrix protein does not contain a conventional nuclear localization sequence but is required for DNA binding and protein self-association. Biochemistry 47:2199-2210.

23. Henderson BR, Eleftheriou A. 2000. A comparison of the activity, sequence specificity, and CRM1-dependence of different nuclear export signals. Exp. Cell Res. 256:213-224.

24. Jin J, et al. 2007. Distinct intracellular trafficking of equine infectious anemia virus and human immunodeficiency virus type 1 Gag during viral assembly and budding revealed by bimolecular fluorescence complementation assays. J. Virol. 81:11226-11235.

25. Jin J, Sturgeon T, Weisz OA, Mothes W, Montelaro RC. 2009. HIV-1 matrix dependent membrane targeting is regulated by Gag mRNA trafficking. PLoS One 4:e6551. doi:10.1371/journal.pone.0006551.

26. Jouvenet $\mathrm{N}$, et al. 2006. Plasma membrane is the site of productive HIV-1 particle assembly. PLoS Biol. 4:e435. doi:10.1371/ journal.pbio.0040435.

27. Jouvenet N, Simon SM, Bieniasz PD. 2009. Imaging the interaction of HIV-1 genomes and Gag during assembly of individual viral particles. Proc. Natl. Acad. Sci. U. S. A. 106:19114-19119.

28. Kaplan AH, Manchester M, Swanstrom R. 1994. The activity of the protease of human immunodeficiency virus type 1 is initiated at the membrane of infected cells before the release of viral proteins and is required for release to occur with maximum efficiency. J. Virol. 68: $6782-6786$.

29. Kemler I, Barraza R, Poeschla EM. 2002. Mapping of the encapsidation determinants of feline immunodeficiency virus. J. Virol. 76: $11889-11903$

30. Kemler I, Meehan A, Poeschla EM. 2010. Live-cell coimaging of the genomic RNAs and Gag proteins of two lentiviruses. J. Virol. 84:63526366.

31. Kosugi S, Hasebe M, Tomita M, Yanagawa H. 2009. Systematic identification of cell cycle-dependent yeast nucleocytoplasmic shuttling proteins by prediction of composite motifs. Proc. Natl. Acad. Sci. U. S. A. 106:10171-10176.

32. Krishnan L, et al. 2010. The requirement for cellular transportin 3 (TNPO3 or TRN-SR2) during infection maps to human immunodeficiency virus type 1 capsid and not integrase. J. Virol. 84:397-406.
33. Kutluay SB, Bieniasz PD. 2010. Analysis of the initiating events in HIV-1 particle assembly and genome packaging. PLoS Pathog. 6:e1001200. doi: 10.1371/journal.ppat.1001200.

34. Lee K, et al. 2010. Flexible use of nuclear import pathways by HIV-1. Cell Host Microbe 7:221-233.

35. Lin YC, Beck Z, Morris GM, Olson AJ, Elder JH. 2003. Structural basis for distinctions between substrate and inhibitor specificities for feline immunodeficiency virus and human immunodeficiency virus proteases. J. Virol. 77:6589-6600.

36. Llano M, et al. 2006. An essential role for LEDGF/p75 in HIV integration. Science 314:461-464.

37. Luttge BG, et al. 2008. Molecular characterization of feline immunodeficiency virus budding. J. Virol. 82:2106-2119.

38. Mariani R, et al. 2000. A block to human immunodeficiency virus type 1 assembly in murine cells. J. Virol. 74:3859-3870.

39. Mullers E, Stirnnagel K, Kaulfuss S, Lindemann D. 2011. Prototype foamy virus gag nuclear localization: a novel pathway among retroviruses. J. Virol. 85:9276-9285.

40. Parent LJ. 2011. New insights into the nuclear localization of retroviral Gag proteins. Nucleus 2:92-97.

41. Poeschla E, Looney D. 1998. CXCR4 is required by a nonprimate lentivirus: heterologous expression of feline immunodeficiency virus in human, rodent, and feline cells. J. Virol. 72:6858-6866.

42. Poeschla E, Wong-Staal F, Looney D. 1998. Efficient transduction of nondividing cells by feline immunodeficiency virus lentiviral vectors. Nat. Med. 4:354-357.

43. Renault N, et al. 2011. A nuclear export signal within the structural Gag protein is required for prototype foamy virus replication. Retrovirology 8:6. doi:10.1186/1742-4690-8-6.

44. Saenz DT, Barraza R, Loewen N, Teo W, Poeschla E. 2006. Production and use of feline immunodeficiency virus (FIV)-based lentiviral vectors, $\mathrm{p}$ 57-74. In Rossi J, Friedman T (ed), Gene transfer: a Cold Spring Harbor Laboratory manual. Cold Spring Harbor Laboratory Press, Cold Spring Harbor, NY.

45. Scheifele LZ, Garbitt RA, Rhoads JD, Parent LJ. 2002. Nuclear entry and CRM1-dependent nuclear export of the Rous sarcoma virus Gag polyprotein. Proc. Natl. Acad. Sci. U. S. A. 99:3944-3949.

46. Scheifele LZ, Kenney SP, Cairns TM, Craven RC, Parent LJ. 2007. Overlapping roles of the Rous sarcoma virus Gag p10 domain in nuclear export and virion core morphology. J. Virol. 81:10718-10728.

47. Scheifele LZ, Ryan EP, Parent LJ. 2005. Detailed mapping of the nuclear export signal in the Rous sarcoma virus Gag protein. J. Virol. 79:87328741.

48. Schliephake AW, Rethwilm A. 1994. Nuclear localization of foamy virus Gag precursor protein. J. Virol. 68:4946-4954.

49. Shaner NC, Steinbach PA, Tsien RY. 2005. A guide to choosing fluorescent proteins. Nat. Methods 2:905-909.

50. Sherer NM, et al. 2011. Evolution of a species-specific determinant within human CRM1 that regulates the post-transcriptional phases of HIV-1 replication. PLoS Pathog. 7:e1002395. doi:10.1371/ journal.ppat.1002395.

51. Swanson CM, Malim MH. 2006. Retrovirus RNA trafficking: from chromatin to invasive genomes. Traffic 7:1440-1450.

52. Swanson CM, Puffer BA, Ahmad KM, Doms RW, Malim MH. 2004 Retroviral mRNA nuclear export elements regulate protein function and virion assembly. EMBO J. 23:2632-2640.

53. Talbott RL, et al. 1989. Nucleotide sequence and genomic organization of feline immunodeficiency virus. Proc. Natl. Acad. Sci. U. S. A. 86:5743-5747.

54. Thys W, et al. 2011. Interplay between HIV entry and transportin-SR2 dependency. Retrovirology 8:7. doi:10.1186/1742-4690-8-7.

55. Tobaly-Tapiero J, et al. 2008. Chromatin tethering of incoming foamy virus by the structural Gag protein. Traffic 9:1717-1727.

56. Zufferey R, Nagy D, Mandel RJ, Naldini L, Trono D. 1997. Multiply attenuated lentiviral vector achieves efficient gene delivery in vivo. Nat. Biotechnol. 15:871-875. 